\title{
latrogenic Narrowing of Inferior Vena Cava after Atrial Septal Defect Closure: The Role of Intraoperative Echocardiography
}

\author{
${ }^{1}$ Shrinivas Gadhinglajkar, ${ }^{2}$ Rupa Sreedhar, ${ }^{3}$ Pravin Lovhale, ${ }^{4}$ Thomas Mathew, ${ }^{5}$ Neeraj Tapdia
}

\begin{abstract}
Single-patch closure of a superior sinus venosus atrial septal defect (SVASD) may be complicated with narrowing of inferior vena cava (IVC). If the pressure gradient across the narrowed portion of superior vena cava (SVC) exceeds $6 \mathrm{~mm} \mathrm{Hg}$, widening of SVC lumen is considered. No such guidelines are available in literature for narrowing of inferior vena cava in cardiac surgical patients. We describe an incident of inferior narrowing of inferior vena cava in a 10-year-old girl, who was operated for closure of an inferior type of ostium secundum ASD (OSASD). There was hepatic venous dilatation accompanied with gradient of $3 \mathrm{~mm} \mathrm{Hg}$ at the junction of inferior vena cava and right atrium (RA). The cardiopulmonary bypass was reestablished and the surgical closure of the septal defect was revised to rectify the problem.
\end{abstract}

Keywords: Atrial septal defect, Inferior vena cava, Transesophageal echocardiography.

How to cite this article: Gadhinglajkar S, Sreedhar R, Lovhale $\mathrm{P}$, Mathew T, Tapdia N. latrogenic Narrowing of Inferior Vena Cava after Atrial Septal Defect Closure: The Role of Intraoperative Echocardiography. J Perioper Echocardiogr 2015;3(2):48-51.

Source of support: Nil

Conflict of interest: None

\section{INTRODUCTION}

Surgical closure of a sinus venosus atrial septal defect (SVASD), situated in close proximity with superior vena cava (SVC), may be complicated with narrowing of inferior vena cava. Usually, a Doppler pressure gradient of $>6 \mathrm{~mm} \mathrm{Hg}$ across the SVC narrowing is considered

\footnotetext{
1,2 Professor, ${ }^{3,5}$ Senior Resident, ${ }^{4}$ Assistant Professor

1,2Department of Anesthesia, Sree Chitra Tirunal Institute for Medical Sciences and Technology, Thiruvananthapuram, Kerala India

${ }^{3}$ Department of Cardiothoracic and Vascular Anesthesia, Sree Chitra Tirunal Institute for Medical Sciences and Technology Thiruvananthapuram, Kerala, India

${ }^{4,5}$ Department of Cardiovascular and Thoracic Surgery, Sree Chitra Tirunal Institute for Medical Sciences and Technology Thiruvananthapuram, Kerala, India
}

Corresponding Author: Shrinivas Gadhinglajkar, Professor Department of Anesthesia, Sree Chitra Tirunal Institute for Medical Sciences and Technology, Thiruvananthapuram, Kerala India, Phone: 04712434053, e-mail: shri@sctimst.ac.in a significant obstruction and it necessitates widening of SVC lumen using an additional patch. ${ }^{1-3}$ Iatrogenic narrowing of inferior vena cava (IVC) after cardiac surgery has been reported rarely in patients with mitral valve replacement ${ }^{4}$ and partially perforated Eustachian valve. ${ }^{5}$ However, how much Doppler pressure gradient would be acceptable across the IVC narrowing in cardiac surgical patients has not been clearly defined. We report an incident of IVC narrowing after the surgical closure of an ostium secundum ASD (OSASD) situated near the IVC, which was detected on intraoperative echocardiography.

\section{CASE REPORT}

A 10-year-old girl, weighing $18 \mathrm{~kg}$, was operated for closure of an OSASD. Preoperative history consisted of recurrent respiratory infections since early childhood. On examination, heart rate and blood pressure were 96/ minute and 110/76 mm Hg respectively. Transthoracic echocardiographic examination revealed a $26 \mathrm{~mm}$ OSASD shunting predominantly left-to-right and absence of IVC rim. The hematological and biochemical parameters were within acceptable limits. She was scheduled for elective surgical closure of the OSASD as the defect was not amenable to device closure due to deficient IVC rim. On the day of the surgery, she was premedicated with oral diazepam $3 \mathrm{mg}$. On arrival in operation room (OR), standard monitors were attached and anesthesia was induced with intravenous fentanyl $100 \mathrm{mcg}$, midazolam $2 \mathrm{mg}$ and propofol $40 \mathrm{mg}$. Vecuronium $3 \mathrm{mg}$ was administered for muscle relaxation before tracheal intubation. Anesthesia was maintained with oxygen-air mixture $\left(\mathrm{FiO}_{2} 0.3\right)$ and isoflurane 0.5 to $1.5 \%$. A triple-lumen cannula was inserted in right internal jugular vein under ultrasound guidance. A pediatric transesophageal echocardiography (TEE) probe was inserted and heart was inspected using an ultrasound system (iE33; Philips Medical Systems, Bothell, WA). The findings on preoperative transthoracic echocardiography were confirmed (Video 1). After heparin administration, cardiopulmonary bypass (CPB) was established and electromechanical quiescence achieved with infusion of antegrade tepid blood cardioplegia. No clear demarcation could be seen between the IVC and left atrium (LA). The ASD was closed directly with sutures, which was followed by closure of right atrium 

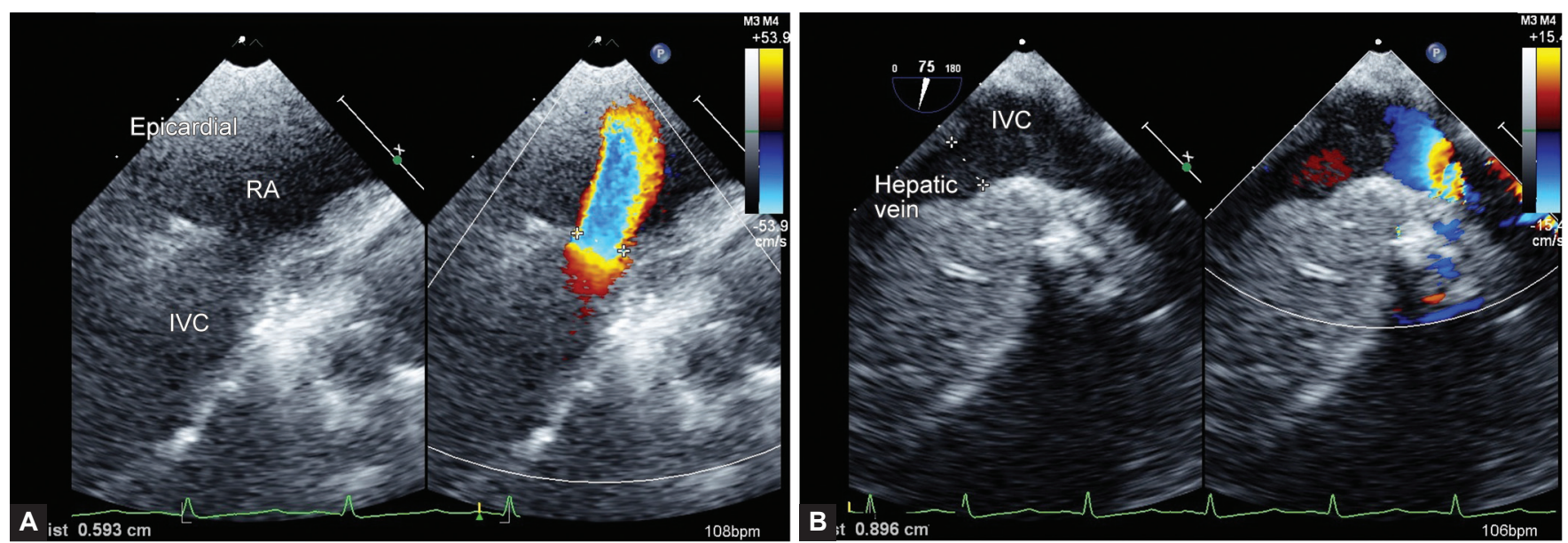

Figs 1A and B: (A) Epicardial echocardiographic examination of IVC-RA junction in a color comparison mode shows narrowing of the junction $(5.9 \mathrm{~mm})$ and $(B)$ hepatic venous inspection after advancing the probe from midesophageal bicaval view reveals hepatic venous diameter of $8.9 \mathrm{~mm}$

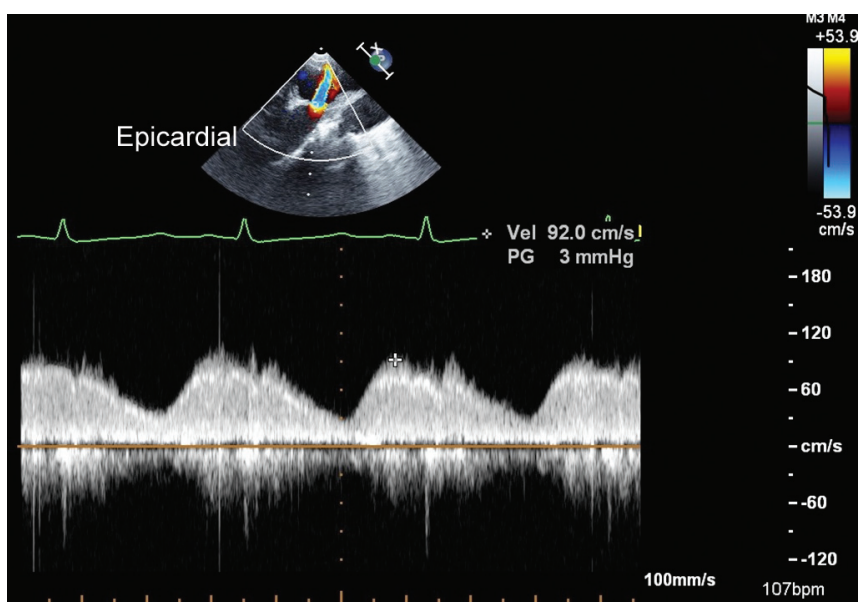

Fig. 2: Epicardial scanning at IVC-RA junction shows peak pressure gradient of $3 \mathrm{~mm} \mathrm{Hg}$

(RA). Patient was weaned from CPB and TEE examination repeated.

After weaning patient from $\mathrm{CPB}$, the ASD patch was examined for any residual leak, rerouting of IVC flow to RA and any evidence of IVC obstruction. On advancing the probe from ME bicaval view, a shelf-like projection was seen at the IVC-RA junction, which was creating flow acceleration and narrowing of the IVC $(5.9 \mathrm{~mm})$. The hepatic vein was dilated $(8.9 \mathrm{~mm})$. Since Doppler beam could not be placed parallel to IVC flow in midesophageal views, we examined the IVC-RA junction in transgastric $\mathrm{RV}$ inflow view. The sector angle was rotated to $155^{\circ}$ and probe was slightly rotated clockwise to bring the IVC-RA junction parallel to the line of Doppler interrogation. However, since it was not optimum, epigastric scanning was performed by placing probe directly over the IVCRA junction (Figs 1A and B, Video 2). Epicardial scanning revealed a gradient of $3 \mathrm{~mm} \mathrm{Hg}$ (Fig. 2). Surgeon recollected that since anatomical boundaries of the IVC and LA were not distinct, the ASD sutures could have been placed low in the IVC. As the IVC lumen became smaller than the dilated hepatic vein, the $\mathrm{CPB}$ was re-established and ASD closure was revised. Patient was weaned from CPB and surgical results were reassessed using echocardiography. The IVC diameter had now increased to $11.2 \mathrm{~mm}$ and hepatic venous size had reduced to $7.8 \mathrm{~mm}$. Postoperative epicardial echo showed a gradient of $1 \mathrm{~mm} \mathrm{Hg}$ across the IVC-RA junction (Figs 3A and B, Video 3). Patient was transferred to intensive care unit (ICU) and artificially ventilated for 2 hours before tracheal extubation. Her postoperative recovery was uneventful.

\section{DISCUSSION}

The inferior sinus venosus ASD should be differentiated from secundum ASD with inferior extension as the course of the surgery will be different. The inferior SVD as a defect originates in the mouth of the IVC and continues directly into the inferoposterior border of the LA, leaving no residual atrial septal tissue at the inferior margin. It is associated with over-riding of IVC and a higher incidence of partial anomalous pulmonary venous return than the secundum ASD. The secundum ASDs with inferior extension typically are positioned in the posteroinferior position of the atrial septum, and a rim of tissue always exists between the ASD and the mouth of the IVC. The over-riding of IVC is absent in these defects. Surgery for inferior SVASD consists of patch closure or construction of a baffle for rerouting pulmonary venous flow and IVC flow to LA and RA respectively. ${ }^{6}$ Iatrogenic complications after the surgical repair of inferior ASD may be attributed to errors in identifying the anatomical structures. Diversion of IVC flow to LA may occur due to mistaken identity of Eustachian valve. ${ }^{78}$ Surgeon had committed error in distinguishing the wall of IVC from that of the LA in our patient due to deficient rim of atrial septal tissue around the ASD near the IVC region. The ASD closure suturing was accidentally extended into the IVC lumen, which 

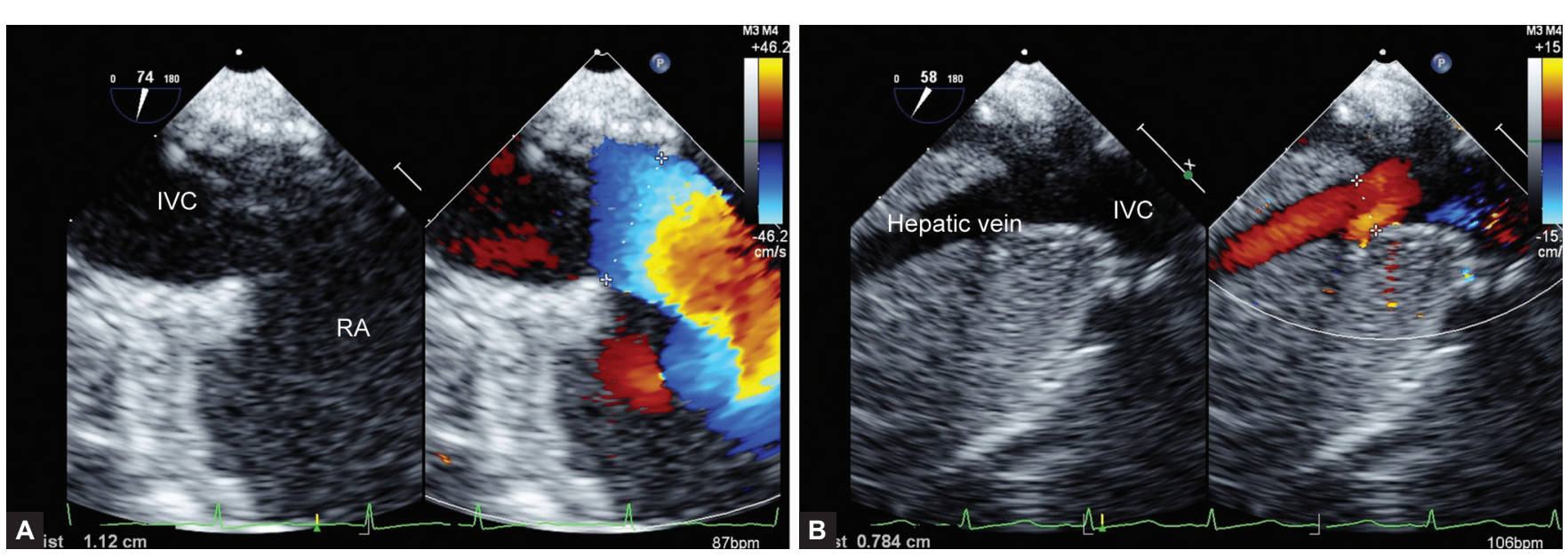

Figs $3 A$ and $B$ : The IVC diameter is increased to $11.2 \mathrm{~mm} \mathrm{(A)} \mathrm{and} \mathrm{hepatic} \mathrm{venous} \mathrm{diameter} \mathrm{reduced} \mathrm{to} 7.8 \mathrm{~mm}(\mathrm{~B})$ in midesophageal views after the surgical revision of ASD

resulted in creating a shelf of tissue within the lumen. Partial obstruction to IVC may increase the proximal IVC pressure. Partial obstruction to the SVC lumen after single-patch closure of superior SVASD is described in the literature. The back-pressure effect of SVC obstruction may lead to cerebral congestion. If the gradient exceeds 5 to $6 \mathrm{~mm} \mathrm{Hg}$, it is advisable to widen the site of SVC narrowing to prevent consequences of venous congestion..$^{1-3}$ The IVC obstruction after the surgical closure of ASD is rare. Hence, there is paucity in the literature on the issue of management of partial IVC obstruction.

The blood flow to the brain is determined by cerebral perfusion pressure, which is the difference in the mean arterial pressure and central venous pressure when the intracranial pressure is normal. Although the rise in the SVC pressure after SVASD closure may obstruct the cerebral venous drainage, it may not cause significant reduction in the cerebral perfusion pressure, as the brain perfusion is determined primarily by the driving mean arterial pressure. Persistent SVC gradient $>15 \mathrm{~mm}$ Hg after SVASD closure may be associated with neurological symptoms like giddiness. ${ }^{3}$ In order to reduce the back-pressure effects of venous obstruction, surgical re-intervention is indicated if the pressure gradient increases $>6 \mathrm{~mm} \mathrm{Hg}$. The pathophysiology of partial obstruction to IVC, however, may differ in this respect from that of the SVC obstruction. Under physiological conditions, $75 \%$ of blood is supplied to liver by the portal vein and remaining $25 \%$ by the hepatic artery. Since the portal venous system operates at a pressure $<10 \mathrm{~mm} \mathrm{Hg}$ and the hepatic artery at the mean arterial pressure, the sinusoidal vessels play a major role in maintaining the balance between the two systems. ${ }^{9}$ The hepatic venous pressure gradient is the difference between the hepatic sinusoidal pressure and the free hepatic venous pressure (FHVP). It represents the gradient between the portal venous pressure and the IVC, which normally ranges between 1 and $5 \mathrm{~mm} \mathrm{Hg}$. The FHVP is usually close to the IVC pressure, the difference being less than $2 \mathrm{~mm} \mathrm{Hg}$. When there is no obstruction between the hepatic vein and the RA, the gradient between these two sites is 1 to $2 \mathrm{~mm}$ $\mathrm{Hg}$. However, this gradient is higher even if there is mild obstruction between these two sites. Thus, any increase in the IVC pressure increases the FHVP, which then increases the hepatic sinusoidal pressure and portal venous pressure..$^{10}$ Apart from liver, other abdominal organs, like gut and kidneys, become vulnerable to the back-pressure effects of the IVC obstruction. The IVC-RA gradient under anesthesia in our patient was $3 \mathrm{~mm} \mathrm{Hg}$; which, however, was likely to increase during exercise. As the hepatic vein became larger than the IVC-RA junction, we decided to proceed with surgical reintervention.

One of the limitations of the 28 standard TEE views recommended by the American Society of Echocardiography is that none of the views is optimum for placing the spectral Doppler beam parallel to the IVC flow. The RA can be visualized from transgastric RV inflow view. Rotating the probe rightward and increasing sector angle beyond $140^{\circ}$ in an off-axis view may bring the IVC-RA junction in focus. However, many times, it may not be possible in this view to maintain the angle of insonation less than $20^{\circ}$ between the Doppler beam and the IVC flow. Epicardial echo has the advantage of obtaining high resolution images and has adequate maneuverability ${ }^{11}$ to place the probe along the long axis of IVC. Hence, it is best suited for measuring the IVC gradient. However, epicardial echocardiography offers a limited visualization of deeper structures like the abdominal portion of the IVC and hepatic vein, which can be ascertained better on TEE in midesophageal views. Hence, TEE and epicardial echo are complimentary to each other during the assessment of the IVC narrowing after cardiac surgery. 


\section{SUMMARY}

We report an incident of iatrogenic narrowing of the IVC after surgical closure of an inferior secundum ASD. Both TEE and epicardial echocardiography are useful monitoring tools in detecting the pathology at IVC-RA junction.

\section{REFERENCES}

1. Russell JL, LeBlanc JG, Deagle ML, Potts JE. Outcome following repair of sinus venosus atrial septal defects in children. Asian Cardiovasc Thorac Ann 2002;10(3):231-234.

2. Gajjar TP, Hiremath CS, Desai NB. Surgical closure of sinus venosus atrial septal defect using a single patch-transcaval repair technique. J Card Surg 2011;26(4):429-434.

3. Iyer AP, Somanrema K, Pathak S, Manjunath PY, Pradhan S, Krishnan S. Comparative study of single- and double-patch techniques for sinus venosus atrial septal defect with partial anomalous pulmonary venous connection. J Thorac Cardiovasc Surg 2007;133(3):656-659.

4. Sharma V, Wasowicz M, Brister S, Karski J, Meineri M. Postoperative transesophageal echocardiography diagnosis of inferior vena cava obstruction after mitral valve replacement. Anesth Analg 2011;113(6):1343-1346.
5. Walpot J, Storm C, Bosmans J, Paelinck BP, Pasteuning WH, Wielenga J, Klazen C, Hokken R, Sorgedrager J, Teeuwen J. Right atrial inflow obstruction of the inferior vena cava due to atrial septal aneurysm and an elongated Eustachian valve. Eur J Echocardiogr 2008;9(6):861-864.

6. Crystal MA, Al Najashi K, Williams WG, Redington AN, Anderson RH. Inferior sinus venosus defect: echocardio-graphic diagnosis and surgical approach. J Thorac Cardiovasc Surg 2009;137(6):349-355.

7. Banka P, Bacha E, Powell AJ, Benavidez OJ, Geva T. Outcomes of inferior sinus venosus defect repair. J Thorac Cardiovasc Surg 2011;142(3):517-522.

8. Jain SA, Pinto R, Dalvi B. Iatrogenic diversion of IVC to left atrium after surgical closure of ASD. Ann Pediatr Cardiol 2012;5(1):72-74.

9. Eipel C, Abshagen K, Vollmar B. Regulation of hepatic blood flow: the hepatic arterial buffer response revisited. World J Gastroenterol 2010;16(48):6046-6057.

10. Kumar A, Sharma P, Sarin SK. Hepatic venous pressure gradient measurement: time to learn. Ind J Gastroenterol 2008;27(2):74-80.

11. ReevesST, Glas KE, Eltzschig H, etal.Guidelines for performing a comprehensive epicardial echocardiography examination: recommendations of the American Society of Echocardiography and the Society of Cardiovascular Anesthesiologists [Review]. J Am Soc Echocardiogr 2007;20(4):427-437. 